\title{
THE BASE SYSTEM: A SCHOOL-WIDE POSITIVE BEHAVIOUR SUPPORT TOOL TO FACILITATE EVIDENCE-BASED DIGITAL INTERVENTION PRACTICES
}

\author{
E. Mariscalco ${ }^{1}$, G. Merlo${ }^{1}$, G. Chiazzese ${ }^{1}$, A. Chifari ${ }^{1}$, S.L. Goei ${ }^{2}$, E. Mangina ${ }^{3}$, A. \\ Mirisola4, I. Giammusso ${ }^{4}$, M. Sanches-Ferreira ${ }^{5}$, P. Denaro ${ }^{1}$, L. Seta ${ }^{1}$ \\ ${ }^{1}$ Consiglio Nazionale delle Ricerche - Istituto per le Tecnologie Didattiche (ITALY) \\ ${ }^{2}$ Vrije Universiteit Amsterdam (NETHERLANDS) \\ ${ }^{3}$ University College of Dublin (IRELAND) \\ ${ }^{4}$ Università degli Studi di Palermo (ITALY) \\ ${ }^{5}$ University of Porto (PORTUGAL)
}

\begin{abstract}
The Positive Behaviour Intervention and Support system is a framework aimed to introduce a change at school-wide level. It promotes a disciplinary system change process, from a reactive punishmentbased strategies of specific student misbehaviours to a proactive system, where different behavioural principles such as the modelling and reinforcement of positive prosocial students' behaviours are applied to improve school values and to create a positive climate. This paper presents the Behavioural Assessment to improve School Environment (BASE) system and the BASE repository. The BASE system supports evidence-based digital intervention practices for stimulating the academic, social, emotional, and behavioural competencies of all students. The BASE repository is a collection of good practices, tools, and instructional contents. Both of the tools are able to support and facilitate, through the use of mobile devices and a web-based responsive system, different prevention and instructional practices at the three-tiers PBS model.

At the first level of prevention (Tier 1) the system allows to the school PBS team to define the Expectation Matrix, a set of positive behaviours grouped according to predefined school values and locations. Moreover, the PBS team members are able to define the list of problem behaviours, classifying them in minor and major. The matrix and the list of minor and major problem behaviours are at the base of the development of a screening tool for identifying behavioural risk problems, the Positive Office Referral and the Office Disciplinary Referral tools. At the target prevention level (Tier 2) the BASE application provides the Check-in/Check-out (CICO) tool, as PBIS recommends. It is addressed to a targeted group of students, resulted unresponsive to the Tier I, and implements the practice of 'Positive reinforcement contingent on meeting behavioural goals' throughout a reward system. At the intensive prevention level (Tier 3 ) the system allows users to perform Functional Behaviour Assessment for students considered unresponsive to Tier I and II and to create customized measurement tools for designing single case studies. The measure can be assigned to the observers able to collect data and organize them in phases. A TAU analysis algorithm is applied to the gathered data for showing the effectiveness of intervention. In the BASE application, each student can access to the system with personal credentials and to visualize his significant progresses into a smart dashboard.

The BASE repository represents a hub for digital resources collection concerning both theoretical and methodological aspects of the PBIS approach. The Internet users interested to know the European experience of the involved partner schools, and to enlarge their knowledge about the principles to implement the PBIS in their own school, can find a first set of multimedia contents, webinars, collection of good practices gathered during the lifespan of the European Erasmus+ BASE project. The repository facilitates the finding of high-quality contents and represents a learning corner and an important knowledge repository for teachers and health professionals to understand and apply this approach.
\end{abstract}

Keywords: PBIS, evidence-based approach, SEBD, behavioural monitoring, web application, responsive design. 


\section{INTRODUCTION}

The Behavioural Assessment to improve School Environment (BASE) project would promote a disciplinary system change in the European schools on the basis of the adoption of Positive Behaviour Support framework. In this framework, the school system shifts from the use of reactive punishmentbased strategies of specific student misbehaviours to a proactive system of promoting positive behaviour. The approach is based on behavioural principles such as the modelling and reinforcement of positive prosocial students' behaviours as school values with the aim to create a positive climate in the behaviour management [1]. A first key element is to improve teachers' abilities in the management of positive behavioural interventions according to the PBIS with the support of evidence-based tools. Three tiers characterize the application of the PBS according to the target users and on the basis of intensity of intervention. The Tier 1 is a school wide practice usually called "universal" experienced by all students and educators with the aim to promote positive behaviours according to a set of identified school expected values. Rules, routines, and physical arrangements are developed and taught by school staff to prevent initial manifestation of problem behaviours.

The Tier 2 is focused on student unresponsive to the Tier 1 intervention and that require a more structured support and intensive feedback in order to reduce the frequency of the problem behaviours. When the students are unresponsive to the first two levels of intervention a function-based Intervention is applied with the aim to identify antecedents and consequences of a problem behaviour and design a tailored intervention for reducing its expression and increasing the occurrence of the desired one. [2]

The BASE application presented in this paper is developed according to the multitier PBS framework and provide functionalities at the three different levels. It promotes a behavioural monitoring based on digital observation practice. The development of a web-based responsive application for monitoring positive behavioural intervention practices allows to:

provide digital tools for facilitating the management and sharing of a lot of behavioural data;

facilitate the collection of data;

reduce the time of the data collection process usually done by hand;

introduce statistical evidence features for evaluating the effectiveness of an intervention. The next section describes the features of the BASE System.

\section{THE BASE SYSTEM}

The BASE application is conceived as a tool for facilitating evidence-based digital intervention practices.

The combination of the BASE system with the BASE repository (a collection of good practices, tools and instructional contents) is able to support and facilitate, through the use of mobile devices and a web-based responsive system, different prevention and instructional practices at the three-tiers PBS model. The figure 1 shows the transversality of the system functionalities in the three tiers. As pieces of a puzzle the functionality are interconnected and properly defined according to their own level.

The green piece represents the Tier 1 implementation of the BASE system and includes tools relating directly or indirectly to any student at school and are both used as a set-up of BASE application and as a support of other functionalities.

The yellow puzzle piece represents the Tier 2 implementation and contains support tools for targeted group of students unresponsive to the intervention of TIER 1 level.

Lastly, the red puzzle piece (Tier 3 ) represents the set of the most specific tools that can be created and customized according to each student's need providing tool for implementing function-based behavioral intervention practices according to a single-case design research method. 


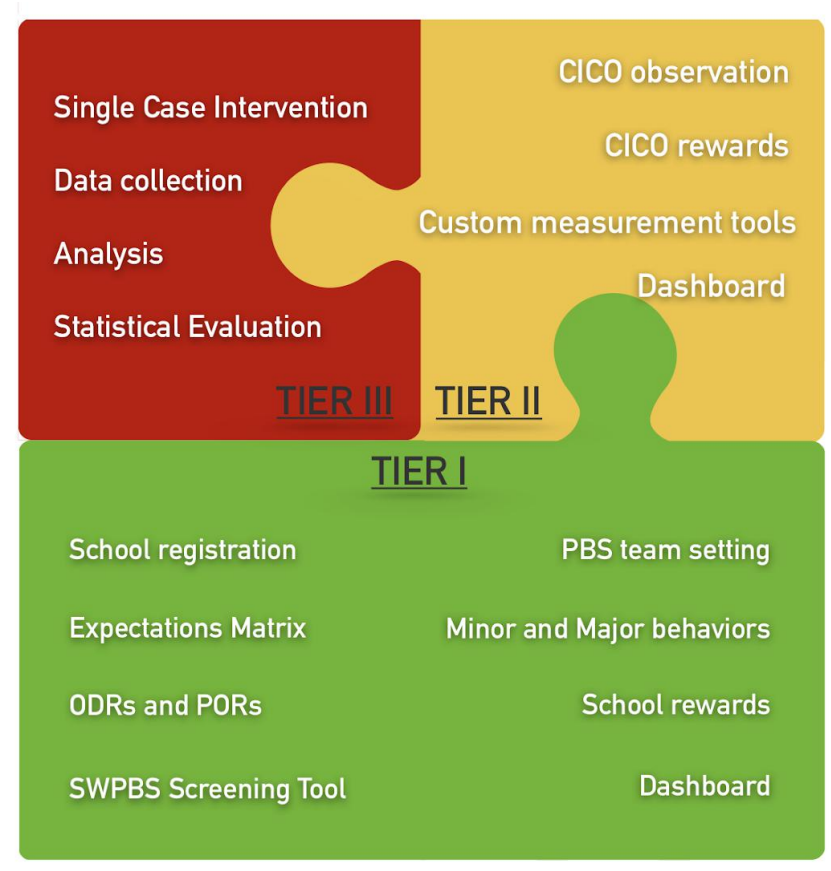

Figure 1. Implementation Tiers of the BASE application

The BASE system has a responsive design structure usable from any type of device.

The architecture chosen for the BASE system is a Client-Server Application, programmed mainly using the PHP Framework Symfony, together with html, CSS and JavaScript tools, while the server part is mainly based on SQL and CouchDB database systems (Fig.2).

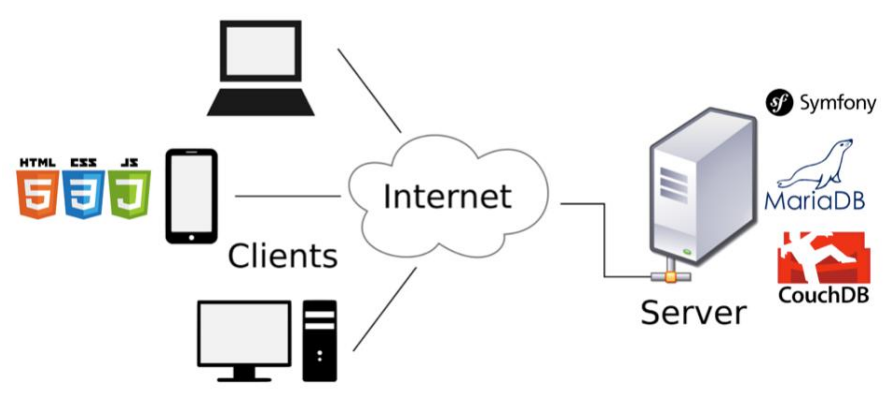

Figure 2 BASE Architecture

The use of the BASE System is differentiated by the definition of functional roles (Fig. 3) inside the school:

[ The School manager registers the school into the BASE system. He can view the list of the registered teachers and assign roles (PBS Team Member and/or teacher coordinator) to them;

- The PBS Team Member creates and manages the Expectations Matrix and the list of minor and major behaviors. S/he can set up several tools, such as the Check-in/Check-out, assign it to observers and visualize the student's data;

w The Teacher Coordinator creates anonymous accounts of his group of students (i.e. classroom);

- The Teacher observes his student's behaviours and access the reports that summarize the student's behavioral data;

= The Student visualizes his data and progresses;

- The Parent observes his child's behaviors and visualize his reports. 


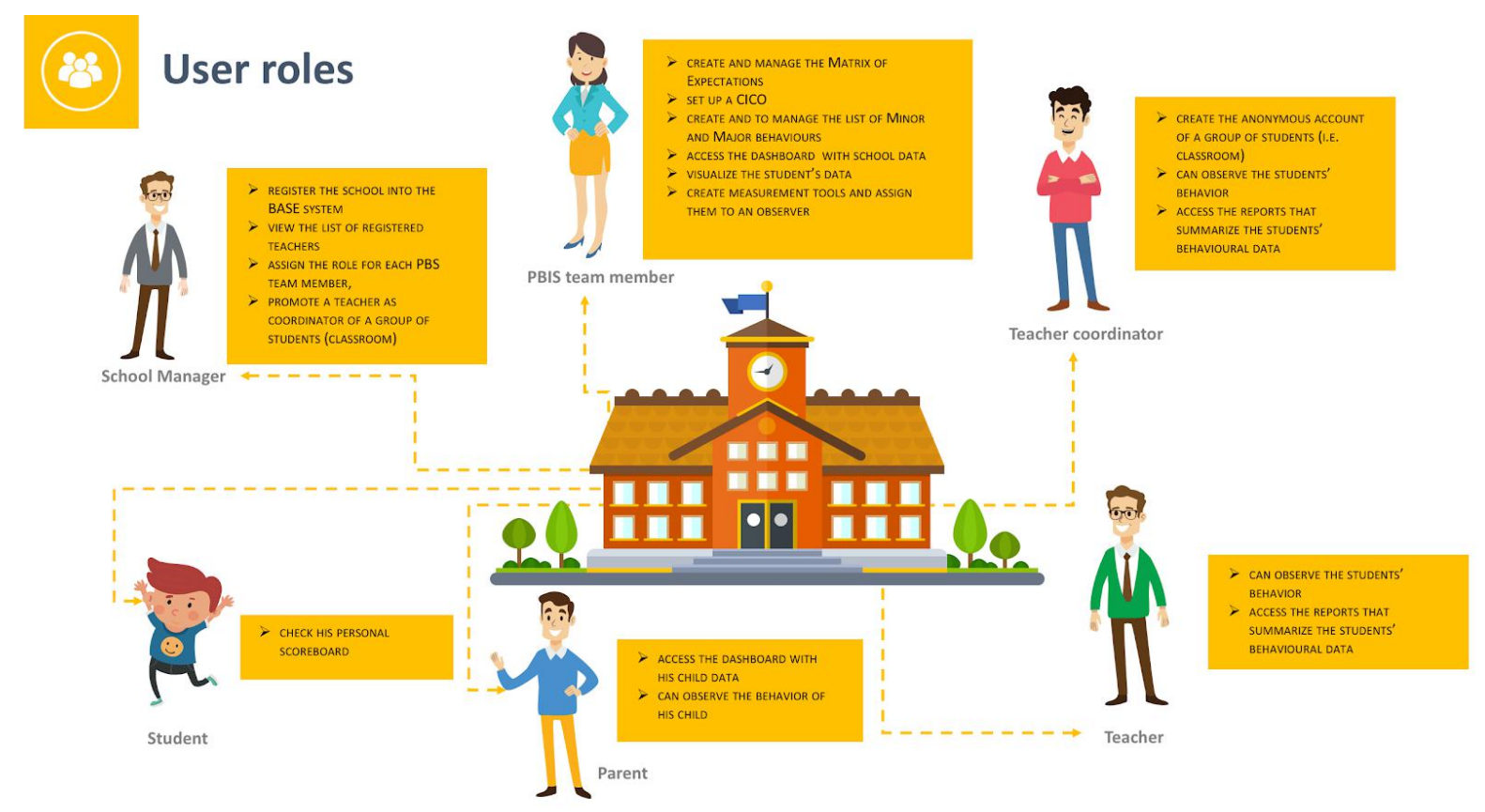

Figure 3. User roles in the BASE application

The privacy of the BASE application is compliant with the General Data Protection Regulation (EU 2016/679). Teachers access the application using their existing accounts provided by companies like Google. The application uses in fact a protocol (Oauth2.0) that allows users to login without inserting the password. Their personal data are stored in the companies' servers that are fully GDPR compliant. For what concerns personal data of students, parents, and school manager they are fully anonymized since they access the application using an alphanumeric code or a QR code. The school is the only responsible of keeping secret the association between the code assigned of the system and the personal data of the student outside of the BASE system.

\subsection{The Tier I features}

The system allows to the school PBS team to define the Expectations Matrix (Fig. 4), a set of positive behaviors grouped according to predefined school values and locations. This matrix presents also a school motto that embodies the ideals and beliefs of the school. Moreover, the PBS team members are encouraged to define the list of problem behaviors, classifying them in minor and major nature. The Expectations Matrix and the list of minor and major problem behaviors are usable in other implemented tools. A list of reward menu associated with points is agreed with students and complete the setup tools available in the BASE application, a list of rewards.

\section{BASE}

Personal Page - School Tools - Measurement Tools *

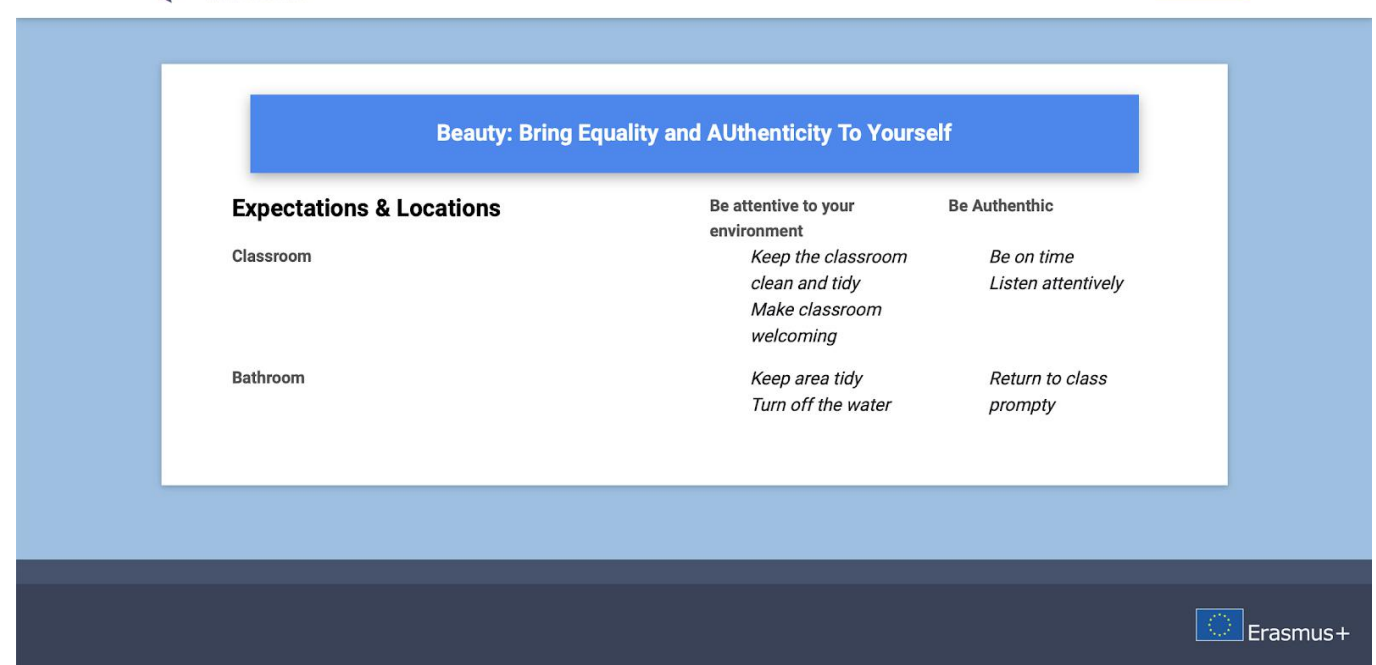

Figure 4. Expectations Matrix sample 
The School Wide Positive Behaviour Support expectations has been implemented as screening tool [3] for identifying behavioural risk problems. This screening tool has a five-points Likert scale to evaluate each student according to the Expectations Matrix values.

The Positive Office Referral and the Office Disciplinary Referral are tools for collecting and monitoring the occurrence and persistence of disruptive behaviors as well as of positive and correct behaviors.

\subsection{The Tier II features}

According to the PBS framework the second level of prevention the system implements the Checkin/Check-out (CICO) tool (Fig.5). It is addressed to a targeted group of students, resulted unresponsive to the other intervention level, and implements the practice of 'Positive reinforcement contingent on meeting behavioral goals' throughout a reward system. The $\mathrm{CICO}$ tool is based on a three-points Likert scale, by which teachers evaluate students everyday during each school period within a CICO session.

At the end of each day, students who overcome their personal goals receive a certain number of points they could spend in the rewards previously described.

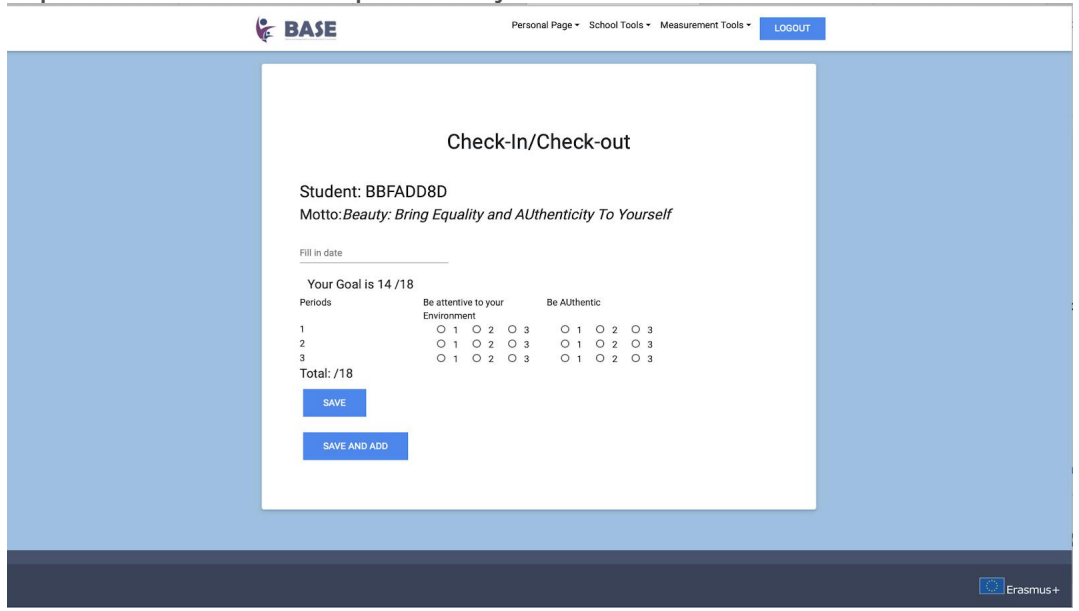

Figure 5. Check-in/Check-out session

The data gathered in Tier I and Tier II are collected and visualized in a smart dashboard with charts, lists and widgets. The teacher of a specific class is able to visualize his students' dashboards (Fig. 6), monitor his data and assign rewards.

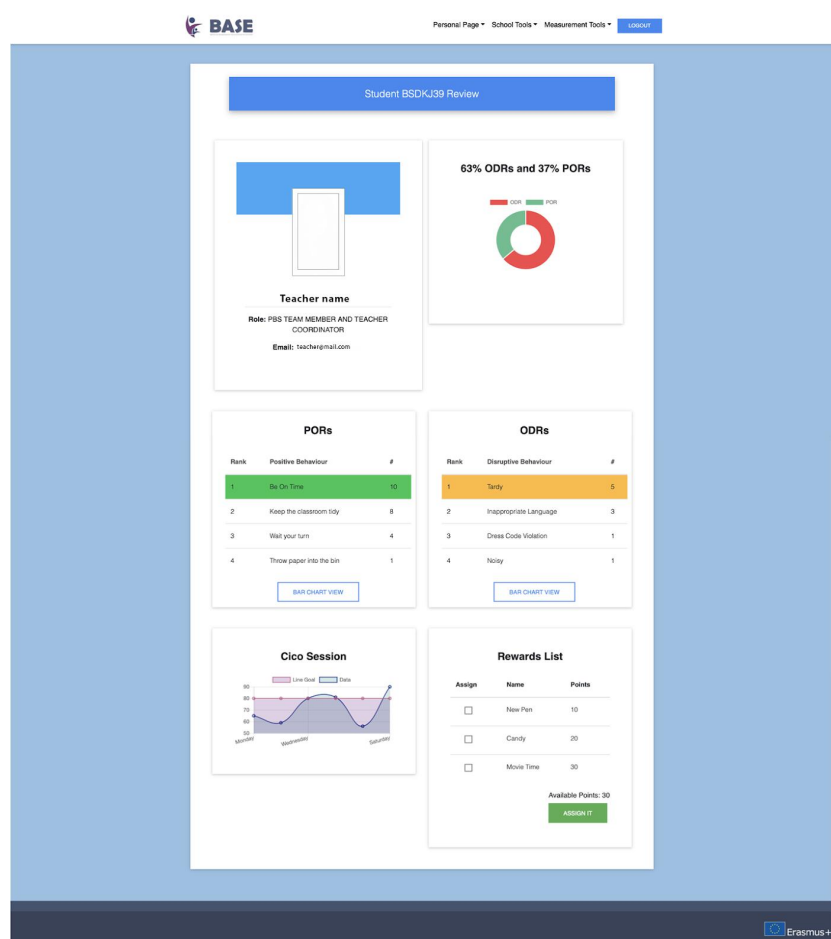

Figure 6. The BASE dashboard 


\subsection{The Tier III features}

The system allows users to perform Functional Behavior Assessment for students considered unresponsive to other intervention and to create customized measurement tools for designing single case studies (TIER III). The measure can be assigned to an observer able to collect data. The collected data will be organized according to the phases of design.

The system adopted an algorithm to analyse the data gathered through the application based on a Monte Carlo study able to choose the better solution among different Tau-U indices of effectiveness of an intervention. TAU-U [4] is a non-parametric method of statistical analysis that combines nonoverlap and trend and computes three different indices by comparing phases of interest. A first index compares two phases (A vs. B, e.g. baseline and intervention) and summarizes information about their nonoverlap. A second index, $A$ vs. $B+$ Trend $B$, adds to the previous information the change in the slope in the $B$ phase. Finally, the third index adds a correction for the phase $A$ trend $(A$ vs. $B+$ Trend $B$ - Trend A). The algorithm is complemented with a decision tree that, by considering bias for each case, suggests the index that better fits with data.

The result of this tool is the evaluation of the well-being status of students and the effectiveness of the intervention and the consequently generation of chart reports.

\section{THE BASE REPOSITORY}

The BASE repository provides to the Internet users high quality contents about the application of Positive Behaviour Support (PBS) at school using the Information Communication Technologies. The BASE repository is created as a reference for the public to have access to research done throughout 5 countries in Europe that are aiming to implement PBS (positive Behaviour Support) in schools.

The contents released as open data will be structured and published online according to the best practices defined by the W3C. The contents are released as Open Educational Resources (OER) according to the following methodology:

the use of an open license for the contents produced (such as Creative Commons);

the use of file formats that are usable by most of the devices on the market;

the subdivision of the materials in tiny reusable modules.

The repository will include the following materials:

data gathered at school through the BASE application about a variety of measures and gathered in many contexts;

the collection of good practices gathered during the project released as Open Educational Resources;

a reasoned collection of multimedia contents.

OMEKA open source content management system for online digital collections has been utilised for the BASE digital repository. As a web application, it allows users to publish and exhibit the collection tree based on the needs of the BASE project as shown in Figure 7 and extend its functionality with themes and plugins.

The features of Omeka has been used for sharing the BASE data collections, teaching materials between BASE users.

Uploaded materials included:

1. methodological notes translated and adapted to each country partner (including demographic data, describing each local experience);

2. video of the interview to school representatives transcribed and translated;

3. documents including behavioural agreements, disciplinary procedures and pictures (e.g., fliers, awards etc.). 


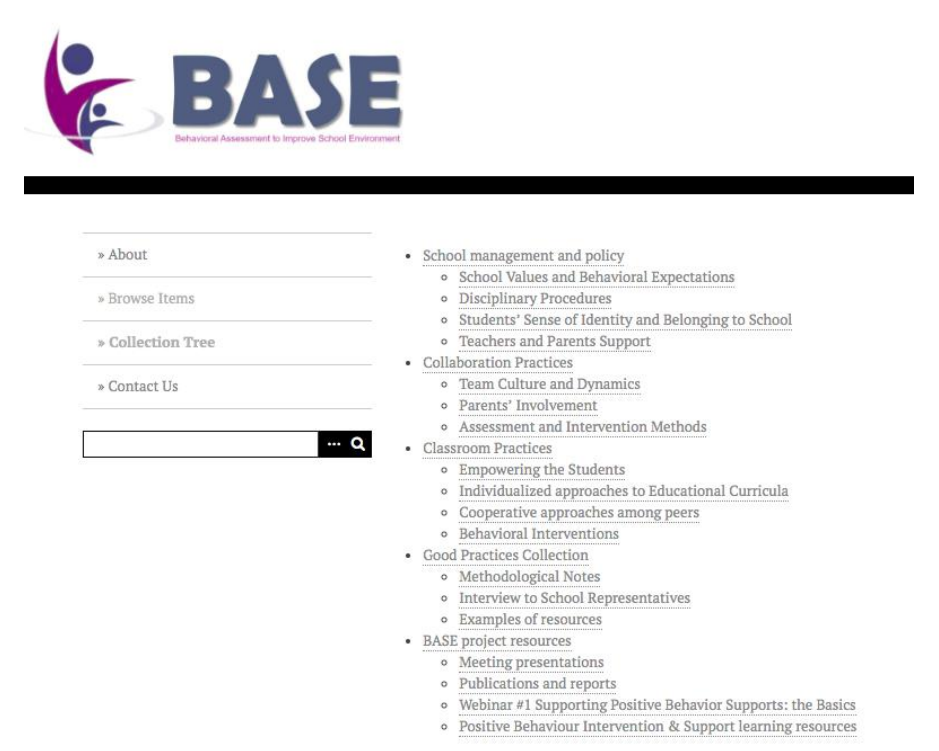

Figure 7. The BASE repository collection

During the initial evaluation of usage and import of files from the users, most of the users $(82.3 \%)$ felt confident using the BASE Repository (Fig. 8).

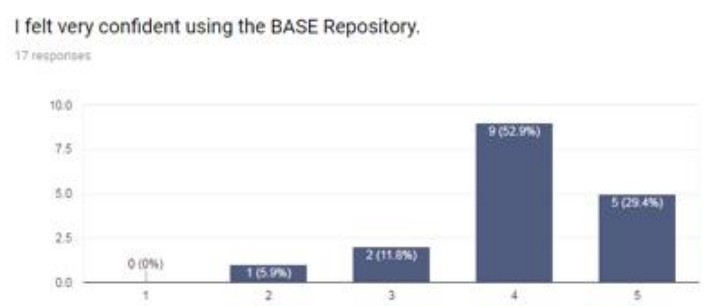

Figure 8. Interest in using the BASE repository

The contents of the repository will be available for at least 5 years at the end of the project, trying to constitute an important knowledge base on the Internet about the application of the PBS in European schools with the support of the BASE application, developed during the project.

\section{CONCLUSIONS}

The BASE (Behavioural Assessment to improve School Environment) project, in response to the European requirement of reforming the whole scholastic disciplinary system identifies a solution in the proven US-born concept of the Positive Behaviour Approach (PBS) and adapts its practical and evidence-based principles to the heterogeneous European school settings. The features of PBS are rooted in the behavioural science and in the practice of Functional Behaviour Analysis (FBA). It works very well in coping with challenging behavioural problems in everyday school life. The BASE innovative web-based software helps school directors and teachers to prevent and effectively face the occurrence of problem behaviours as well as teachers' burnout and students' disengagement. The BASE project ambition is to disseminate across Europe the philosophy underpinning the proven US concept of PBS approach throughout the data collected with the BASE web application. The data collected have been imported in the BASE repository, which provides the Internet users with high quality contents about the application of PBS at school using the Information Communication Technologies. BASE project takes advantage from the considerable experience of an interdisciplinary consortium for the identification, adoption, implementation, and monitoring of the research-validated practices and in helping educators to understand and improve their teaching styles and approaches to meet the emerging challenges as and when they arise. 


\section{ACKNOWLEDGEMENTS}

BASE has received funding from the Erasmus+ Program: KA2 - Cooperation for innovation and Exchange of Good Practices KA202 - Strategic Partnership for school education Project no. 2017-1RO01-KA201-037460.

\section{REFERENCES}

[1 ] N.A. Gage, G. Sugai, T.J. Lewis, and S. Brzozowy, "Academic achievement and school-wide positive behavior supports," Journal of Disability Policy Studies, vol. 25, no. 4, pp. 199-209, 2015

[2] G. Sugai, and R. H. Horner. "Defining and describing schoolwide positive behavior support". In Handbook of positive behavior support (Sailor et al. eds.), pp. 307-326., Boston: Springer, 2009

[3] M.D. Burke, J.L. Davis, S. Hagan-Burke, Y.H. Lee and M.S. Fogarty "Using SWPBS expectations as a screening tool to predict behavioral risk in middle school". Journal of Positive Behavior Interventions, vol. 16 no. 1, pp. 5-17, 2014.

[4] R.L. Parker, K.J. Vannest, J.L. Davis, and S.B. Sauber, "Combining Nonoverlap and Trend for Single-Case Research: Tau-U,” Behavior Therapy, vol.42, pp. 284-299, 2011. 